\title{
Long-term case-fatality rate of nontuberculous mycobacterial disease in people living with HIV
}

Jingjing $\mathrm{Hu}^{1,2+} \mathbb{D}$, Ling $\mathrm{Gu}^{2+}$, Yueming Shao ${ }^{2}$, Renfang Zhang ${ }^{2}$, Tangkai $\mathrm{Qi}^{2}$, Jianjun Sun ${ }^{2}$, Zhenyan Wang ${ }^{2}$, Wei Song ${ }^{2}$, Yang Tang ${ }^{2}$, Jiangrong Wang ${ }^{2}$, Shuibao Xu², Junyang Yang ${ }^{2}$, Yinzhong Shen², Li Liu²*, Jun Chen ${ }^{2^{*}}$ and Hongzhou Lu ${ }^{1,3^{*}}$

\begin{abstract}
Background: Few data are available regarding the long-term case-fatality rate (CFR) among people living with HIV $(P L W H)$ with nontuberculous mycobacteria (NTM) disease. The aim of this study is to analyze the long-term CFR in patients with NTM disease and to identify risk factors for their death.

Methods: A retrospective cohort study of 379 cases of microbiologically confirmed NTM disease in PLWH was conducted from January 1, 2012, to December 31, 2020, in Shanghai, China. We used Kaplan-Meier survival analysis and the log-rank test to compare the long-term CFR in patients with disseminated NTM (DNTM) and localized NTM disease. Univariate Cox proportional hazards regression analysis and a stepwise Cox proportional hazards regression model were used to estimate the predictors of long-term CFR.

Results: The cohort was followed up for a median of 26 months. The total CFR was $15.7 \%$ by one year and increased to $22.6 \%$ at 5 years after the diagnosis of NTM disease. The 5 -year CFR of PLWH with DNTM was significantly higher than that of PLWH with localized NTM (26.7\% vs $19.6 \%$ for DNTM and localized NTM disease, respectively). Older age [hazard ratio $(H R)=1.04,95 \%$ confidence interval $(C): 1.02-1.06, P<0.001$ ], comorbidity (HR=2.05, 95\% Cl: 1.21-3.49, $P<0.01)$, DNTM ( $H R=2.08,95 \% C l: 1.17-3.68, P<0.05)$, and HIV viral load $(H R=1.32,95 \% C l: 1.12-1.55, P<0.001)$ were all independent risk factors for long-term CFR. In the subgroup analysis, time to culture positivity was negatively correlated with CFR in patients with DNTM ( $H R=0.90,95 \% \mathrm{Cl}: 0.82-0.98, P<0.05)$.
\end{abstract}

Conclusions: NTM was associated with a high long-term CFR in PLWH. Further approaches to prevent NTM disease in PLWH are urgently needed.

Keywords: HIV/AIDS, Nontuberculous mycobacteria, Case-fatality rate, Risk factor

\footnotetext{
*Correspondence: liuli@shphc.org.cn; qtchenjun@163.com;

luhongzhou@fudan.edu.cn

${ }^{\dagger}$ Jingjing Hu and Ling Gu contributed equally to this work

${ }^{1}$ School of Public Health, Bengbu Medical College, Bengbu 233000,

Anhui, China

2 Department of Infectious Diseases and Immunology, Shanghai Public

Health Clinical Center, Fudan University, 2901 Caolang Road, Jinshan

District, Shanghai 201508, China

Full list of author information is available at the end of the article
}

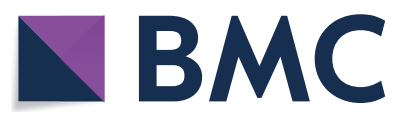

Ther(s) 2022. Open Access This article is licensed under a Creative Commons Attribution 4.0 International License, which permits use, sharing, adaptation, distribution and reproduction in any medium or format, as long as you give appropriate credit to the original author(s) and the source, provide a link to the Creative Commons licence, and indicate if changes were made. The images or other third party material in this article are included in the article's Creative Commons licence, unless indicated otherwise in a credit line to the material. If material is not included in the article's Creative Commons licence and your intended use is not permitted by statutory regulation or exceeds the permitted use, you will need to obtain permission directly from the copyright holder. To view a copy of this licence, visit http://creativecommons.org/licenses/by/4.0/. The Creative Commons Public Domain Dedication waiver (http://creativeco mmons.org/publicdomain/zero/1.0/) applies to the data made available in this article, unless otherwise stated in a credit line to the data. 


\section{Graphical Abstract}

Data collection: Three hundred seventy-nine cases of microbiologically confirmed NTM disease in PLWH was included from January 1, 2012, to December 31, 2020 , in Shanghai, China.

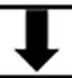

Survival analysis: Cox proportional hazards regression analysis and Kaplan-Meier survival analysis were used to estimate the predictors of long-term CFR.

Result: Older age, comorbidity, DNTM and HIV viral load were independent risk factors for long-term CFR in PLWH with NTM.

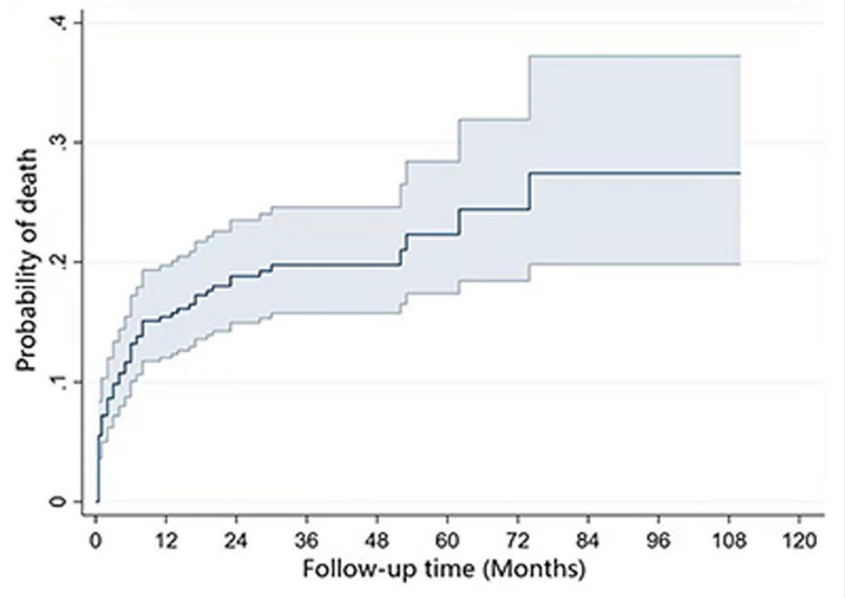

we conducted this study to analyze the long-term CFR in PLWH with NTM and to identify risk factors for death.

\section{Methods}

\section{Study subjects}

A retrospective analysis was performed on the clinical data of PLWH with NTM in Shanghai Public Health Clinical Center from January 1, 2012, to December 31, 2020. The inclusion criteria were as follows: (1) HIV-1 infection confirmed by Western blotting; (2) patients had at least one specimen with positive mycobacterial culture and negative for MPB64, or the mycobacterial sequencing results were NTM; (3) physicians deemed NTM to be an etiology of the diseases but not colonization. The exclusion criteria were patients younger than 18 years. years and the highest incidence in those with CD4 cell count $<50$ cells $/ \mathrm{mm}^{3}$ (5,300/100,000 person-years) [4].

In the preantiretroviral therapy era, case fatality rates (CFRs) were high for NTM and even higher for DNTM, with an annual CFR of 71\% [5]. Since the antiretroviral therapy (ART) era, AIDS has become a chronic disease, which has led to a significant increase in life expectancy in PLWH, and the incidence of disseminated Mycobacterium avium complex (DMAC) has declined significantly from 65.3/1,000 in 1992 to 2.0/1,000 in 2015 [6]. However, despite the availability of effective ART, the CFR for NTM remains high, with a CFR of $69 \%$ at 1 year and $27 \%$ at 3 years after the diagnosis of DMAC [7].

Modern population-based estimates of the long-term survival of HIV-infected patients with NTM are lacking. Studies have indicated that the long-term survival of PLWH with tuberculosis (TB) is lower than that of nonTB patients [8]. We hypothesized that long-term CFR would also be elevated in PLWH with NTM. Therefore,

\section{Study design}

The following characteristics were recorded: sex, age, behavioral risk factors (such as current or former smoking, alcohol abuse), comorbidities, opportunistic infections, $\mathrm{CD}^{+}{ }^{+} \mathrm{T}$ cell count, HIV viral load, ART regimen, NTM treatment, symptoms and the time from specimen culture initiation to positive report. Furthermore, we registered the high-resolution computed tomography (CT) scan. All abnormal CT scans were reported and divided into seven categories: (1) lymphadenopathy only; (2) lymphadenopathy with nodules; (3) lymphadenopathy with cavities; (4) lymphadenopathy with cavities and nodules; (5) nodules only; (6) cavities only; (7) and consolidation only. We recorded the laboratory data of all patients, including T-SPOT results, blood cell counts, hemoglobin levels, erythrocyte sedimentation rates, C-reactive protein levels and renal and liver function. 
The diagnosis of DNTM was defined as a positive culture for mycobacterium from blood, cerebrospinal fluid, bone marrow, or biopsy of a sterile site or infection involving two or more noncontiguous body sites. Comorbidities were defined based on the Charlson Comorbidity Index $[9,10]$, which includes 19 major disease categories, including risk factors and potential prognostic factors for PLWH with NTM. Comorbidity data included diabetes, hypertension, cancer, chronic lung disease and other diseases. AIDS-defining opportunistic infections other than NTM infection were also recorded. Baseline CD4 ${ }^{+}$ $\mathrm{T}$ cell count and HIV viral load were defined using the test results at admission or the closest record at admission. Anti-NTM medication use was defined as drug use for at least 2 weeks.

For the prognosis analysis, survival time was defined as the time from the beginning of definitive diagnosis of NTM to death, loss to follow-up, or the end of follow-up (December 31, 2020). Patients were followed up by telephone after discharge from the hospital. The outcome was all-cause mortality during the follow-up period.

The study was approved by the Ethics Committee of Shanghai Public Health Clinical Center (Ethics approval number: 2020-Y112-01). Informed consent was waived because of the retrospective design of the study.

\section{Statistical analysis}

SPSS statistics 25.0 (IBM, Armonk, NY, USA) and Stata 16.0 (StataCorp LP, College Station, TX, USA) were used for statistical analysis. The Shapiro-Wilk test was used to test whether the data conformed to a normal distribution. Normally distributed data are reported as the means and standard deviation (mean $\pm \mathrm{SD}$ ). Nonnormally distributed data are presented as the medians and interquartile range (IQR). Categorical variables were summarized with frequency counts and presented as a rate (\%). The $\chi^{2}$ test, $t$ test and Fisher's exact test were used to test for statistically significant differences. Kaplan-Meier survival analysis and log-rank test were used to compare long-term CFR in patients with DNTM and localized NTM disease. Univariate Cox proportional hazards regression analysis and stepwise Cox proportional hazards regression models were used to estimate the predictors of long-term CFR. $P<0.05$ indicated statistical significance. In these analyses, hazard ratios were combined with $95 \%$ confidence intervals.

\section{Results}

\section{Clinical characteristics of the study population}

Three hundred seventy-nine patients were included. Of these, $93.7 \%$ were male, and the median age was 38.0 (IQR: $30.0-50.0$ ) years. In total, $7.4 \%$ of the patients were current or former smokers, and $2.6 \%$ consumed alcohol.
One hundred thirteen patients $(29.8 \%)$ had comorbidities, and 136 patients (35.9\%) had opportunistic infections. In addition, the median $\mathrm{CD} 4^{+} \mathrm{T}$ cell count was 23.0 (IQR: 6.0-73.8) cells/ $\mu \mathrm{l}$, and the median HIV viral load was 4.84 (IQR: $1.9-5.5) \log 10$ copies/ml. Two hundred ninety-four patients $(77.6 \%)$ received ART prior to anti-NTM therapy, and the median time from initiation of ART to initiation of anti-NTM therapy was 31.0 (IQR: 4.0-127.0) months (Table 1).

The most frequently reported symptom was fever (63.4\%). Cough was reported in $44.1 \%$ of all cases. A total of $22.8 \%$ reported HIV wasting syndrome, $18.3 \%$ reported abdominal pain and/or diarrhea, $12.9 \%$ reported central nervous system symptoms such as headache and dizziness, and half of these patients had a combination of cryptococcal meningitis. The remaining $11.6 \%$ of patients had skin manifestations, such as rashes (Table 1). For each year from 2013 to 2020, DNTM accounted for almost half of the total number of NTM in PLWH (Fig. 1). Among the first reported positive specimens, sputum accounted for $60.7 \%$; blood accounted for $23.5 \%$; and stool accounted for $6.9 \%$; while the rest were puncture fluid $(4.0 \%)$, bronchoalveolar lavage or bronchial lavage fluid $(1.3 \%)$, pleural effusion $(0.8 \%)$, cerebrospinal fluid $(0.8 \%)$, bone marrow $(0.8 \%)$, urine $(0.5 \%)$, hydroperitoneum $(0.3 \%)$, abdominal abscess $(0.3 \%)$ and secretions from ruptured skin $(0.3 \%)$. The median time to culture positivity was 13.9 (IQR: 9.5-23.5) days.

Three hundred twenty-six (86.0\%) patients received CT scans. Of the 326 patients, 148 (45.4\%) were described as having lymphadenopathy. The most common morphology was mediastinal lymphadenopathy $(128 / 148,86.5 \%)$ (Table 2).

Treatment for NTM diseases consists of a multidrug regimen and a long course of therapy. Anti-NTM medication for patients included macrolides, levofloxacin/ moxifloxacin, ethambutol, rifampicin/rifabutin, and linezolid (Table 1), which lasted for 9-12 months. Almost all of the enrolled patients received ART.

\section{Survival analysis}

After a median of 26 months of follow-up, 69 patients (18.2\%) died, and 48 (12.7\%) were lost to follow-up. In $52.2 \%$ of patients, the follow-up period exceeded 2 years. The life table method showed an overall CFR of $15.7 \%$ at 1 year, $19.0 \%$ at 2 years, $20.0 \%$ at 3 years, $22.6 \%$ at 5 years, and $27.9 \%$ at 7 years. Univariate Cox regression analysis indicated that the following parameters were statistically significant for survival: older age, HIV viral load, ART before NTM treatment, comorbidity, linezolid and DNTM (Table 3). The probability of death in PLWH with NTM increased with time (Fig. 2). 
Table 1 Baseline characteristics of PLWH with NTM infection

\begin{tabular}{|c|c|}
\hline & $n(\%)$ \\
\hline \multicolumn{2}{|l|}{ General information } \\
\hline Total number of patients & 379 \\
\hline Male sex & $355(93.7 \%)$ \\
\hline Age [years], Median (IQR) & $38.0(30.0-50.0)$ \\
\hline Smoking (current or former) & $28(7.4 \%)$ \\
\hline Alcoholism & $10(2.6 \%)$ \\
\hline Comorbidity & $113(29.8 \%)$ \\
\hline Opportunistic infection & $136(35.9 \%)$ \\
\hline Cryptococcosis & $35(25.7 \%)$ \\
\hline Cytomegalovirus & $28(20.6 \%)$ \\
\hline Digestive tract fungal infections & $23(16.9 \%)$ \\
\hline Pneumocystis pneumonia & $12(8.8 \%)$ \\
\hline Talaromyces marneffei & $11(8.1 \%)$ \\
\hline Kaposi's Sarcoma & $8(5.9 \%)$ \\
\hline Herpes zoster & $5(3.7 \%)$ \\
\hline Mycobacterium tuberculosis & $4(2.9 \%)$ \\
\hline Salmonella infection & $3(2.2 \%)$ \\
\hline Pulmonary aspergillosis & $2(1.5 \%)$ \\
\hline Bacterial pneumonia & $2(1.5 \%)$ \\
\hline Cerebral toxoplasmosis & $2(1.5 \%)$ \\
\hline Progressive multifocal leukoencephalopathy & $1(0.7 \%)$ \\
\hline \multicolumn{2}{|l|}{ Clinical manifestations } \\
\hline Fever & $236(63.4 \%)$ \\
\hline Cough & $164(44.1 \%)$ \\
\hline HIV wasting syndrome & $81(22.8 \%)$ \\
\hline Abdominal pain and diarrhea & $68(18.3 \%)$ \\
\hline Central nervous system symptoms & $48(12.9 \%)$ \\
\hline Rash & $43(11.6 \%)$ \\
\hline \multicolumn{2}{|l|}{ HIV-related indicators } \\
\hline $\mathrm{CD}^{+} \mathrm{T}$ cell count [cells/MI], Median (IQR) & $23(6.0-73.8)$ \\
\hline HIV viral load [log 10 copies/ml], Median (IQR) & $4.8(1.9-5.5)$ \\
\hline ART before NTM treatment & $294(77.6 \%)$ \\
\hline ART to anti-NTM time, Median (IQR) & $31(4.0-127.0)$ \\
\hline \multicolumn{2}{|l|}{ NTM treatment } \\
\hline Macrolides & $280(73.9 \%)$ \\
\hline Levofloxacin/Moxifloxacin & $248(65.4 \%)$ \\
\hline Ethambutol & $317(83.6 \%)$ \\
\hline Rifampicin/Rifabutin & $248(65.4 \%)$ \\
\hline Linezolid & $23(6.1 \%)$ \\
\hline
\end{tabular}

PLWH people living with HIV, NTM nontuberculous mycobacteria, IQR interquartile range, HIV human immunodeficiency virus, $A R T$ antiretroviral therapy

Considering that sex, smoking, alcoholism, opportunistic infection, $\mathrm{CD}_{4}^{+} \mathrm{T}$ cell count, and time to culture positivity were also important risk factors, these factors and all parameters that were statistically significant in the univariate analysis were included in a multivariate Cox proportional hazards model. The results showed a hazard ratio of 2.05 [95\% confidence interval $(C I): 1.21-3.49$, $P<0.01]$ for patients with comorbidities compared with those without comorbidities. The hazard ratio caused by older age was 1.04 (95\% CI: $1.02-1.06, P<0.001)$. High levels of HIV viral load were statistically significant, with a hazard ratio of 1.32 (95\% CI: 1.12-1.55, $P<0.001$ ). DNTM was significantly correlated with poor survival outcomes $(\mathrm{HR}=2.08,95 \% \mathrm{CI}: 1.17-3.68, P<0.05)$ (Table 3). Kaplan-Meier analysis also revealed that the long-term CFR of the DNTM group was significantly higher than that of the localized infection group (Fig. 3). Surprisingly, patients not treated with linezolid had a significantly longer survival time than those treated with linezolid (HR = 4.71, 95\% CI: 2.25-9.83, $P<0.001$ ).

In addition, we performed a stratified analysis by baseline $\mathrm{CD} 4^{+} \mathrm{T}$ cell count. Patients with $\mathrm{CD} 4^{+} \mathrm{T}$ cell counts $>50$ cells $/ \mu$ l had CFRs of $7.9 \%, 12.1 \%$, and $17.9 \%$ at 1,3 , and 7 years, respectively. Older age $(\mathrm{HR}=1.09$, 95\% CI: $1.04-1.14, P<0.001)$ and DNTM $(\mathrm{HR}=3.52$, 95\% CI: 1.01-12.28, $P<0.05)$ were independent prognostic factors. Patients with $\mathrm{CD} 4^{+} \mathrm{T}$ cell counts $\leq 50$ cells $/ \mu \mathrm{l}$ had CFRs of $19.9 \%, 24.4 \%$ and $32.7 \%$ at 1,3 and 7 years, respectively. Comorbidities $(\mathrm{HR}=2.14,95 \% \mathrm{CI}$ : 1.19-3.89, $P<0.05)$ and linezolid usage $(\mathrm{HR}=2.97,95 \%$ $C I: 1.34-6.58, P<0.01)$ were independent prognostic factors. ART before NTM treatment was more beneficial for patients $(\mathrm{HR}=0.46,95 \%$ CI: $0.25-0.84, P<0.05)$.

In the subgroup analysis for patients with DNTM, time to culture positivity was negatively correlated with CFR (HR $=0.90,95 \%$ CI: 0.84-0.96, $P<0.01$ ). The longer the time to a positive culture of the specimen, the lower the number of NTMs, thus favoring survival. Older age $(\mathrm{HR}=1.05,95 \% \mathrm{CI}: 1.02-1.08, P<0.01)$, comorbidity $(H R=2.38,95 \% C I: 1.14-4.96, P<0.05)$ and linezolid usage $(\mathrm{HR}=3.39,95 \% C I$ : $1.43-8.02, P<0.01)$ remained independent risk factors for long-term CFR (Table 4).

\section{Discussion}

Our study demonstrated that the long-term CFR of PLWH with NTM is high, despite these patients receiving treatment or even recovering. Further analysis revealed that the long-term CFR for disseminated infections was higher than that for those without disseminated infections. Older age, comorbidity, HIV viral load, DNTM and linezolid usage were independent prognostic factors for PLWH with NTM. Although the CD4 $4^{+} \mathrm{T}$ cell count was not significant in the multivariate analysis, we found that patients with $C D 4^{+} \mathrm{T}$ cell counts $\leq 50$ cells/ $\mu \mathrm{l}$ had a higher CFR. These patients were more likely to acquire DNTM. For patients with DNTM, the time to culture positivity was negatively correlated with CFR.

Collins et al. studied patients with HIV/AIDS from 1992 to 2015 [7]. Despite effective ART, they found 


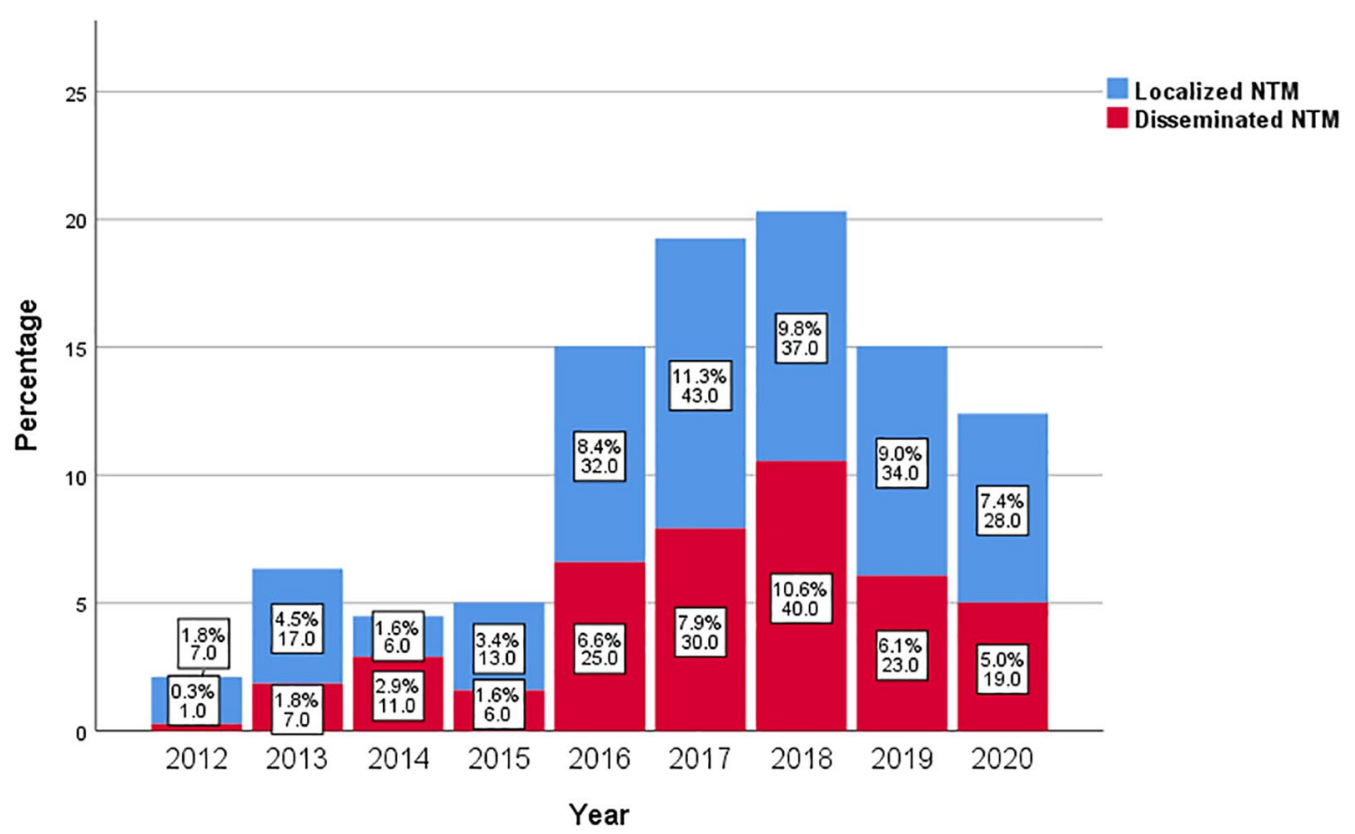

Fig. 1 Stacked bar charts of PLWH with disseminated NTM and with localized NTM disease. (The data showed in the charts is percentage and number). PLWH people living with HIV, NTM nontuberculous mycobacteria

Table 2 Radiology findings of PLWH with NTM infection

\begin{tabular}{|c|c|c|}
\hline & & $n(\%)$ \\
\hline Lymphadenopathy & & $148(45.4 \%)$ \\
\hline \multicolumn{3}{|l|}{ Morphology } \\
\hline & Mediastinal Lymphadenopathy & $128(86.5 \%)$ \\
\hline & Hilar lymphadenopathy & $42(28.4 \%)$ \\
\hline & Retroperitoneal lymph & $40(27.0 \%)$ \\
\hline & Axillary lymph nodes & $20(13.5 \%)$ \\
\hline & Supraclavicular/infraclavicular lymph nodes & $8(5.4 \%)$ \\
\hline & Celiac lymph node & $8(5.4 \%)$ \\
\hline & Pelvic lymph nodes & $1(0.7 \%)$ \\
\hline \multicolumn{3}{|l|}{ Categories } \\
\hline & Lymphadenopathy only & $111(75.0 \%)$ \\
\hline & Lymphadenopathy with nodules & $23(15.5 \%)$ \\
\hline & Lymphadenopathy with cavities & $10(6.8 \%)$ \\
\hline & Lymphadenopathy with cavities and nodules & $4(2.7 \%)$ \\
\hline Nodules & & $33(10.1 \%)$ \\
\hline Cavities & & $14(4.3 \%)$ \\
\hline Consolidation & & $3(0.9 \%)$ \\
\hline Other infectious lesions & & $107(32.8 \%)$ \\
\hline No obvious abnormalities & & $21(6.4 \%)$ \\
\hline
\end{tabular}

Total of 326 patients received CT scans. PLWH people living with HIV, NTM nontuberculous mycobacteria, HIV human immunodeficiency virus

that DMAC infection was associated with a significant increase in CFR in the years following diagnosis. This is similar to the results in our study. Another Japanese study also showed that DNTM was significantly associated with CFR, and the median baseline $\mathrm{CD} 4^{+} \mathrm{T}$ cell count was significantly lower in nonsurvivors than in survivors [11]. However, their sample size of 24 was not very convincing, and further studies are needed. 
Table 3 Hazard ratio in univariate analysis and multivariate analysis

\begin{tabular}{|c|c|c|c|c|c|c|}
\hline & \multicolumn{3}{|c|}{ Univariate analysis } & \multicolumn{3}{|c|}{ Multivariate analysis } \\
\hline & Hazard ratio & $95 \% \mathrm{Cl}$ & $P$ & Hazard ratio & $95 \% \mathrm{Cl}$ & $P$ \\
\hline Age & 1.02 & $1.01-1.04$ & 0.015 & 1.04 & $1.02-1.06$ & 0.001 \\
\hline Comorbidity & 2.01 & $1.24-3.23$ & 0.005 & 2.05 & $1.21-3.49$ & 0.008 \\
\hline DNTM & 1.81 & $1.12-2.90$ & 0.015 & 2.08 & $1.17-3.68$ & 0.012 \\
\hline HIV viral load & 1.25 & $1.07-1.46$ & 0.006 & 1.32 & $1.12-1.55$ & 0.001 \\
\hline Linezolid & 3.64 & $1.85-7.15$ & 0.001 & 4.71 & $2.25-9.83$ & 0.001 \\
\hline Sex & 1.59 & $0.50-5.04$ & 0.435 & - & - & 0.697 \\
\hline Smoking & 0.86 & $0.31-2.35$ & 0.764 & - & - & 0.411 \\
\hline Alcoholism & 1.30 & $0.32-5.29$ & 0.719 & - & - & 0.592 \\
\hline Opportunistic infection & 0.98 & $0.60-1.61$ & 0.946 & - & - & 0.411 \\
\hline $\mathrm{CD}^{+} \mathrm{T}$ cell count & 1.00 & $1.00-1.00^{\mathrm{a}}$ & 0.243 & - & - & 0.795 \\
\hline ART before NTM treatment & 0.53 & $0.32-0.89$ & 0.015 & - & - & 0.221 \\
\hline Time to culture positivity & 0.98 & $0.96-1.01$ & 0.125 & - & - & 0.232 \\
\hline
\end{tabular}

Sex, age, smoking, alcoholism, comorbidity, opportunistic infection, CD4 ${ }^{+}$T cell count, HIV viral load, ART before NTM treatment, linezolid, DNTM and time to culture positivity were added to the model using stepwise procedures

-: blank (In the multivariate analysis, SPSS 25.0 did not show $95 \% \mathrm{Cl}$ and hazard ratio for variables that were not statistically significant); ${ }^{\text {a }}$ 95\% $\mathrm{Cl}$ for hazard ratio of CD4 ${ }^{+} \mathrm{T}$ cell count: $0.996-1.001$

HIV human immunodeficiency virus, NTM nontuberculous mycobacteria, DNTM disseminated nontuberculous mycobacteria, ART antiretroviral therapy

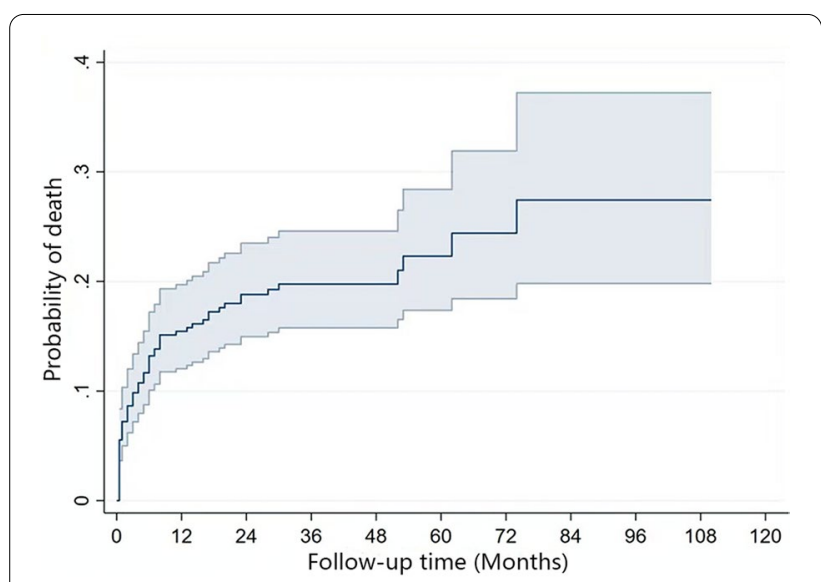

Fig. 2 Probability of death (95\% confidence interval) among PLWH with NTM. PLWH people living with HIV, NTM nontuberculous mycobacteria

Data on the impact of NTM on long-term CFR in PLWH are limited, but studies in HIV-negative cohorts have also found high long-term CFR in NTM survivors. Typically, factors such as older age and comorbidities have been reported to be associated with poor prognosis. A systematic review gave an overall estimate of 5-year CFR from NTM pulmonary disease studies. Despite the high heterogeneity of the enrolled studies, the pooled estimate of 5-year all-cause mortality of the 9035 patients was 27\% (95\% CI: 21.3-37.8) [12]. Predictors of CFR that were consistent across studies included male sex, presence of comorbidities and older age of patients [12]. Since the vast majority of PLWH in our cohort were male, there was a gender skew; thus, our data are not quite applicable to the general population.

Several studies have compared long-term CFR among PLWH after completing TB treatment to those without TB [8]. The 5-year CFR for patients who completed TB treatment was $10.2 \%$ compared to patients without TB (5.6\%) [8]. In our study, the 5-year CFR for NTM was $22.6 \%$, which appears to be more than twice as high as that of TB patients. Chiang et al. also reported that PLWH with DMAC $(n=58)$ had a three times higher 1 -year CFR than those with TB $(n=98)(48.3 \%$ vs $16.3 \%)$ [13]. This implies that the long-term prognosis of NTM in PLWH is of critical importance.

Behavioral factors (such as smoking and alcoholism) $[14,15]$ and various respiratory or nonrespiratory comorbidities [16] increase the risk of acquiring NTM and may partially account for the increased long-term CFR among NTM survivors. Multiple studies in largely HIVnegative populations have documented structural lung defects and impaired pulmonary function after NTM infection, and many studies have found a strong association with increased CFR between a history of NTM and chronic obstructive pulmonary disease and bronchiectasis [17-21]. Recently, Mourad et al. found that the expected survival was reduced by approximately 4 years for a diagnosis of NTM lung disease without comorbidity and by 8.6 years for a diagnosis of NTM lung disease with comorbidity [22]. Among HIV-negative patients with 


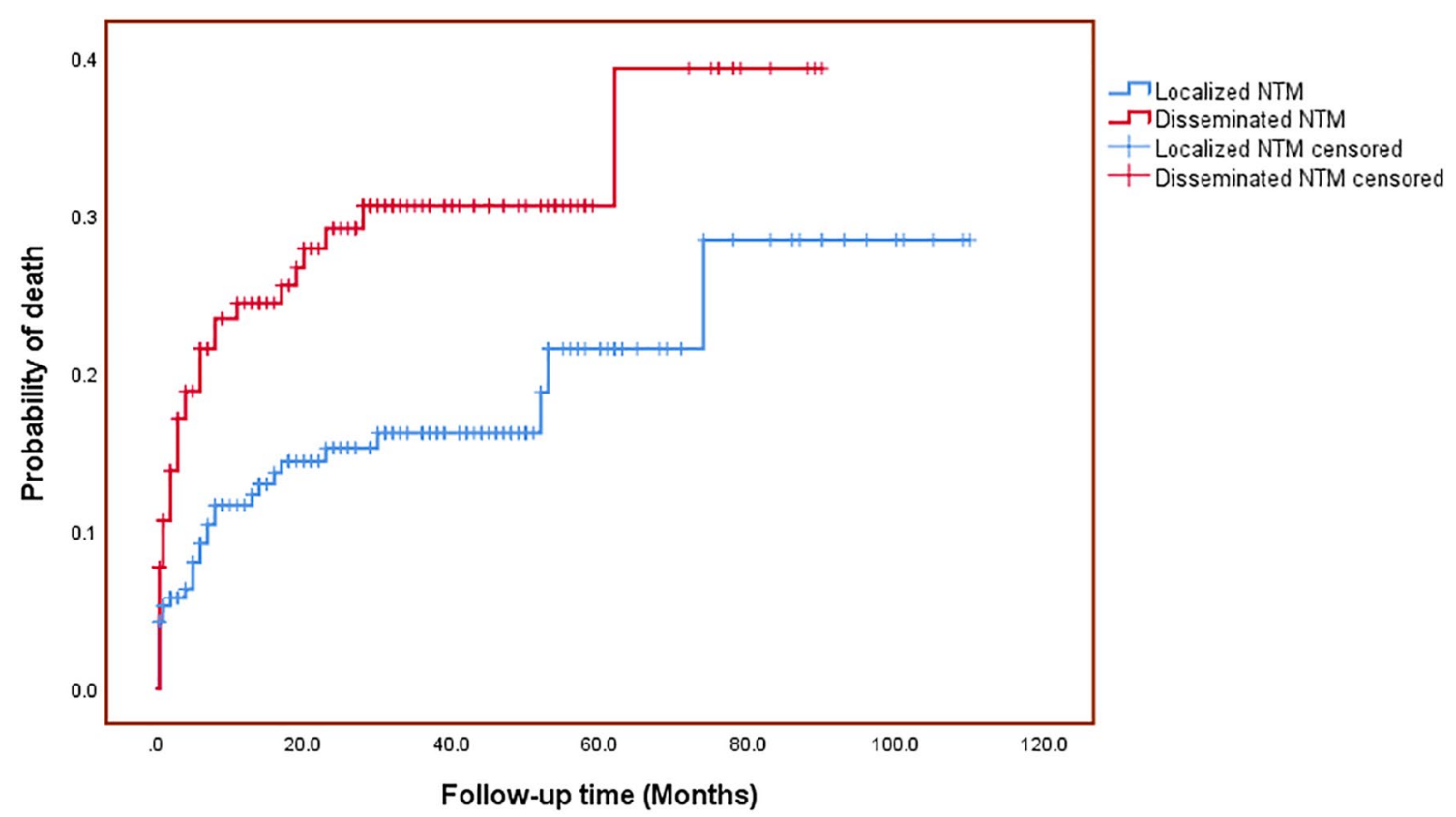

Fig. 3 Probability of death among PLWH with disseminated NTM and localized NTM disease. PLWH people living with HIV, NTM nontuberculous mycobacteria

Table 4 Hazard ratio in multivariate analysis of PLWH with DNTM

\begin{tabular}{llll}
\hline & Hazard ratio & $\mathbf{9 5 \% ~ C l}$ & $\boldsymbol{P}$ \\
\hline Age & 1.05 & $1.02-1.08$ & 0.004 \\
Comorbidity & 2.38 & $1.14-4.96$ & 0.021 \\
Linezolid & 3.39 & $1.43-8.02$ & 0.006 \\
Time to culture positivity & 0.90 & $0.84-0.96$ & 0.002 \\
\hline
\end{tabular}

Sex, age, smoking, alcoholism, comorbidity, opportunistic infection, $\mathrm{CD}^{+} \mathrm{T}$ cell count, HIV viral load, ART before NTM treatment, linezolid, DNTM and time to culture positivity were added to the model using stepwise procedures

HIV human immunodeficiency virus, $A R T$ antiretroviral therapy, $P L W H$ people living with HIV, DNTM disseminated nontuberculous mycobacteria

NTM with and without comorbidities, the 5-year CFR after diagnosis was $44.9 \%$ and $25.0 \%$, respectively [22].

The high occurrence of disease relapse and increasing drug resistance may lead to an increased CFR. A multicenter study showed that $9.5 \%$ of patients with NTM pulmonary infection experienced multiple episodes, with $24.8 \%$ of them suffering from relapsing infections caused by the same NTM species [23]. An observational retrospective study from Italy revealed that $35.3 \%$ of patients had unsuccessful treatment outcomes, including discontinuation of therapy $(13.5 \%)$, recurrence $(11.2 \%)$, reinfection $(5.3 \%)$, treatment failure $(4.1 \%)$ and relapse $(1.2 \%)$ [24]. The treatment of treatment-refractory NTM cases or patients with drug-resistant NTM isolates remains challenging. This may indicate a poor prognosis and high CFR. In clinical practice, patients with NTM infections caused by more resistant species may use linezolid, as their conditions are more severe. This may partially explain why using linezolid was associated with poor outcome in our study.

In addition, NTM may cause persistent inflammation and immune activation, which may increase susceptibility to HIV infection, promote HIV viral replication, and accelerate the progression of HIV disease [25]. As shown in a previous study, 79\% (19/24) of PLWH with DNTM had immune reconstitution syndrome, suggesting difficulty in the management of DNTM [11]. Therefore, clinicians should pay high attention to DNTM in PLWH. However, the diagnosis of NTM infection in PLWH is difficult, as the available methods are limited. Notably, nearly half of the patients had abnormal CT imaging in our study, most commonly mediastinal lymphadenopathy. This may give the physician a clue in treating PLWH suspected of NTM infection.

There are some limitations in our study. First, this study was conducted at a single center, and our results may not be generalizable to other regions due to its retrospective nature. Second, we did not use the American Thoracic Society criteria for NTM lung disease. Third, no further species identification was available for most patients. Several studies have found that different mycobacterium species were not significant for 
CFR analysis [26, 27]. However, a 15-year follow-up study of 1445 patients with NTM pulmonary disease showed that the accurate identification of the species or subspecies of the NTM pathogen is very important in the prognosis [28]. Data from Canada also showed that NTM disease was associated with higher rates of death for all species combined and for most individual species [29]. Therefore, further research on species identification is needed. Furthermore, although the sensitivity of MPB64 is high, a very small percentage of patients may still have a false-negative result [30]. It is possible that a few patients enrolled were due to TB.

\section{Conclusions}

To our knowledge, this is the largest study to date that evaluates the long-term CFR and associated prognostic factors for NTM in PLWH in the modern ART era. Longterm CFR for NTM in PLWH is high. Older age, comorbidity and DNTM are independent prognostic factors for NTM. These findings highlight the critical importance of PLWH with NTM and suggest that PLWH with a history of NTM may require closer long-term follow-up.

\begin{abstract}
Abbreviations
CFR: Case-fatality rate; HIV: Human immunodeficiency virus; PLWH: People living with HIV; NTM: Nontuberculous mycobacteria; DNTM: Disseminated nontuberculous mycobacteria; MAC: Mycobacterium avium Complex; DMAC: Disseminated Mycobacterium avium complex; ART: Antiretroviral therapy; TB: Tuberculosis; CT: Computed tomography; SD: Standard deviation; IQR: Interquartile range.
\end{abstract}

\section{Acknowledgements}

Not applicable.

\section{Authors' contributions}

$\mathrm{HL}, J \mathrm{C}$ and LL conducted the study conception and design. JH and LG collected the data of patients. JH and JC analyzed and interpreted the data. $\mathrm{JH}$ wrote the manuscript. YS made grammatical revisions to the manuscript. $J C$ and $H L$ critically revised and finally approved the manuscript. RZ, TQ, JS, ZW, WS, YT, JW, SX, JY and YS supervised the project. All authors read and approved the final manuscript.

\section{Funding}

This work was supported by the Shanghai Commission of Science and Technology (20MC1920100 and 21Y11901200), Shanghai key Infectious Disease Project (shslczdzk01102), Shanghai Municipal Health Commission (GWV-10.1-XK02), the development fund for Shanghai talents (2020089) and the Shanghai "Rising stars of Medical Talent"Youth Development Program [No. 2019-72].

\section{Availability of data and materials}

The datasets used and/or analyzed during the current study are available from the corresponding author on reasonable request.

\section{Declarations}

\section{Ethical approval and consent to participate}

The study was approved by the Ethics Committee of Shanghai Public Health Clinical Center. (Ethics approval number: 2020-Y112-01). Informed consent was waived because of the retrospective design of the study.

\section{Consent for publication}

Not applicable.

\section{Competing interests}

The authors declare that they have no competing interests.

\section{Author details}

${ }^{1}$ School of Public Health, Bengbu Medical College, Bengbu 233000, Anhui, China. ${ }^{2}$ Department of Infectious Diseases and Immunology, Shanghai Public Health Clinical Center, Fudan University, 2901 Caolang Road, Jinshan District, Shanghai 201508, China. ${ }^{3}$ Shenzhen Third People's Hospital, The Second Affiliated Hospital of Southern University of Science and Technology, Shenzhen 518000, China.

Received: 6 December 2021 Accepted: 28 January 2022

Published online: 07 February 2022

\section{References}

1. Agizew T, Basotli J, Alexander H, Boyd R, Letsibogo G, Auld A, et al. Higherthan-expected prevalence of non-tuberculous mycobacteria in HIV setting in Botswana: implications for diagnostic algorithms using Xpert MTB/RIF assay. PLoS One. 2017;12(12):e189981.

2. Rivero-Lezcano OM, Gonzalez-Cortes C, Mirsaeidi M. The unexplained increase of nontuberculous mycobacteriosis. Int J Mycobacteriol. 2019;8(1):1-6.

3. Lapinel NC, Jolley SE, Ali J, Welsh DA. Prevalence of non-tuberculous mycobacteria in HIV-infected patients admitted to hospital with pneumonia. Int J Tuberc Lung Dis. 2019;23(4):491-7.

4. Varley CD, Ku JH, Henkle E, Schafer SD, Winthrop KL. Disseminated nontuberculous mycobacteria in HIV-infected patients, Oregon, USA, 2007-2012. Emerg Infect Dis. 2017;23(3):533-5.

5. Moore RD, Chaisson RE. Natural history of opportunistic disease in an HIV-infected urban clinical cohort. Ann Intern Med. 1996;124(7):633-42.

6. Chen H, Clifford DB, Deng L, Wu K, Lee AJ, Bosch RJ, et al. Peripheral neuropathy in ART-experienced patients: prevalence and risk factors. J Neurovirol. 2013;19(6):557-64.

7. Collins LF, Clement ME, Stout JE. Incidence, long-term outcomes, and healthcare utilization of patients with human immunodeficiency virus/ acquired immune deficiency syndrome and disseminated Mycobacterium avium complex from 1992-2015. Open Forum Infect Dis. 2017;4(3):x120.

8. Koenig SP, Kim A, Shepherd BE, Cesar C, Veloso V, Cortes CP, et al. Increased mortality after tuberculosis treatment completion in persons living with human immunodeficiency virus in Latin America. Clin Infect Dis. 2020;71(1):215-7.

9. Charlson ME, Pompei P, Ales KL, MacKenzie CR. A new method of classifying prognostic comorbidity in longitudinal studies: development and validation. J Chronic Dis. 1987;40(5):373-83.

10. Quan H, Li B, Couris CM, Fushimi K, Graham P, Hider P, et al. Updating and validating the Charlson comorbidity index and score for risk adjustment in hospital discharge abstracts using data from 6 countries. Am J Epidemiol. 2011;173(6):676-82

11. Kobayashi T, Nishijima T, Teruya K, Aoki T, Kikuchi Y, Oka S, et al. High mortality of disseminated non-tuberculous mycobacterial infection in HIV-infected patients in the antiretroviral therapy era. PLoS ONE. 2016;11(3):e151682.

12. Diel R, Lipman M, Hoefsloot W. High mortality in patients with Mycobacterium avium complex lung disease: a systematic review. BMC Infect Dis. 2018;18(1):206.

13. Chiang $\mathrm{CH}$, Lee GH, Chiang TH, Tang PU, Fang CT. Disseminated Mycobacterium avium complex infection as a differential diagnosis of tuberculosis in HIV patients. Int J Tuberc Lung Dis. 2020;24(9):922-7.

14. Yeligar SM, Chen MM, Kovacs EJ, Sisson JH, Burnham EL, Brown LA. Alcohol and lung injury and immunity. Alcohol. 2016;55:51-9.

15. Agusti A, Hogg JC. Update on the pathogenesis of chronic obstructive pulmonary disease. N Engl J Med. 2019;381(13):1248-56.

16. Lee H, Myung W, Lee EM, Kim H, Jhun BW. Mortality and prognostic factors of nontuberculous mycobacterial infection in Korea: a populationbased comparative study. Clin Infect Dis. 2021;72(10):e610-9. 
17. Balavoine C, Andrejak C, Marchand-Adam S, Blanc FX. Relationships between COPD and nontuberculous mycobacteria pulmonary infections. Rev Mal Respir. 2017;34(10):1091-7 (in French).

18. Flume PA, Chalmers JD, Olivier KN. Advances in bronchiectasis: endotyping, genetics, microbiome, and disease heterogeneity. Lancet. 2018;392(10150):880-90.

19. Berra G, Plojoux J, Soccal PM, Janssens JP. Identification of non-tuberculous mycobacteria in COPD patients undergoing lung volume reduction: more frequent than expected? Respiration. 2019;98(3):279-80.

20. Pyarali FF, Schweitzer M, Bagley V, Salamo O, Guerrero A, Sharifi A, et al. Increasing non-tuberculous mycobacteria infections in veterans with COPD and association with increased risk of mortality. Front Med (Lausanne). 2018:5:311.

21. McShane PJ, Naureckas ET, Tino G, Strek ME. Non-cystic fibrosis bronchiectasis. Am J Respir Crit Care Med. 2013;188(6):647-56.

22. Mourad A, Baker AW, Stout JE. Reduction in expected survival associated with nontuberculous mycobacterial pulmonary disease. Clin Infect Dis. 2021;72(10):e552-7.

23. Huang HL, Cheng MH, Lu PL, Shu CC, Wang JY, Wang JT, et al. Epidemiology and predictors of NTM pulmonary infection in Taiwan - a retrospective, five-year multicenter study. Sci Rep. 2017;7(1):16300.

24. Aliberti S, Sotgiu G, Castellotti P, Ferrarese M, Pancini L, Pasat A, et al. Reallife evaluation of clinical outcomes in patients undergoing treatment for non-tuberculous mycobacteria lung disease: a ten-year cohort study. Respir Med. 2020;164:105899.

25. Havlir DV, Torriani FJ, Schrier RD, Huang JY, Lederman MM, Chervenak KA, et al. Serum interleukin-6 (IL-6), IL-10, tumor necrosis factor (TNF) alpha, soluble type II TNF receptor, and transforming growth factor beta levels in human immunodeficiency virus type 1-infected individuals with Mycobacterium avium complex disease. J Clin Microbiol. 2001;39(1):298-303.

26. Longworth SA, Blumberg EA, Barton TD, Vinnard C. Non-tuberculous mycobacterial infections after solid organ transplantation: a survival analysis. Clin Microbiol Infect. 2015;21 (1):43-7.

27. Wetzstein N, Hügel C, Wichelhaus TA, Hogardt M, Eickmeier O, KüpperTetzel CP, et al. Species distribution and clinical features of infection and colonisation with non-tuberculous mycobacteria in a tertiary care centre, central Germany, 2006-2016. Infection. 2019;47(5):817-25.

28. Jhun BW, Moon SM, Jeon K, Kwon OJ, Yoo H, Carriere KC, et al. Prognostic factors associated with long-term mortality in 1445 patients with nontuberculous mycobacterial pulmonary disease: a 15-year follow-up study. Eur Respir J. 2020;55(1):1900798.

29. Marras TK, Campitelli MA, Lu H, Chung H, Brode SK, Marchand-Austin A, et al. Pulmonary nontuberculous mycobacteria-associated deaths, Ontario, Canada, 2001-2013. Emerg Infect Dis. 2017;23(3):468-76.

30. Raj A, Singh N, Gupta KB, Chaudhary D, Yadav A, Chaudhary A, et al. Comparative evaluation of several gene targets for designing a multiplexPCR for an early diagnosis of extrapulmonary tuberculosis. Yonsei Med J. 2016;57(1):88-96.

Ready to submit your research? Choose BMC and benefit from:

- fast, convenient online submission

- thorough peer review by experienced researchers in your field

- rapid publication on acceptance

- support for research data, including large and complex data types

- gold Open Access which fosters wider collaboration and increased citations

- maximum visibility for your research: over 100M website views per year

At BMC, research is always in progress.

Learn more biomedcentral.com/submissions 